\title{
Nachhaltigkeit, Feministische Ökonomie und die Frage nach der Macht der Leerstellen
}

\author{
Die feministische Ökonomie gibt Blicke auf \\ Leerstellen frei und stellt das klassische Denken \\ auf den Prüfstand. Obwohl sie in einigen wirt- \\ schaftspolitischen Diskursen aufgegriffen wurde, \\ braucht es mehr Konzepte aus der feministischen \\ Ökonomie. Es braucht ein neues ökonomisches \\ Narrativ. Von Deborah Ruggieri
}

W ho cooked Adam Smith's Dinner?“ fragt Katrine Marçal und bringt damit die feministische Kritik an der „Mainstream Ökonomie“ auf den Punkt (Marcal 2016). Es geht um Ein- und Ausschlüsse, um Messbarkeit in Verbindung mit dem scheinbar unumstößlich Faktischen, um das Primat der vorherrschenden Ökonomie, die trotz Kritik immer noch als „alternativlos“ definiert wird (Keen 2011). Es geht um die Macht von Menschenbildern beziehungsweise Glaubenssätzen in der Ökonomie, die sich auch in Natur- und Geschlechterverhältnissen widerspiegeln. Sie werden oft nicht erwähnt. Die feministische Ökonomie legt einen Blick auf Leerstellen frei, die die vorherrschende Ökonomie immer wieder infrage stellen. Hier gibt es eine Schnittstelle zu vielen ökologischen Bewegungen, die das herkömmliche Primat der neoklassischen Ökonomie genauso kritisch analysieren und sogenannte „Selbstverständlichkeiten“ hinterfragen.

Je nach Denkschule aus der feministischen Ökonomie kann sich das Verständnis von Wirtschaftskreisläufen, die Definition von Arbeit, zugrunde liegende Marktlogiken und das Verständnis dessen, wie die Gesellschaft arbeiten, wirtschaften und leben soll, verändern. Die bisherigen Modelle und Annahmen müssen überholt werden, um zukunftsfähig zu sein. Deswegen zurück zu Adam Smith's Dinner. Wer kochte es denn nun und wieso ist das für eine feministische Ökonomie relevant? Die Antwort auf diese Frage ist einfach - seine Mutter. Sie unterstützte den berühmten Nationalökonomen, der als Begründer der klassischen Nationalökonomie gilt, zu seinen Lebzeiten (Raworth 2018).

Diese Frage bringt auch das Entscheidende auf den Punkt: Es geht um das „Nicht-Sichtbare“ oder das nicht sichtbar gemachte in der Ökonomie. Kochen oder Putzen - sich um das leibliche Wohl kümmern ist symptomatisch für Pflege und Sor- gearbeiten, sogenannte „Care“-Tätigkeiten. In klassischen ökonomischen Theorien werden sie als selbstverständlich gesehen, sind nur peripher relevant oder gar nichts „wert“. Und das ist der Kern der Problematik. Denn wenn bestimmte Tätigkeiten als „natürlich“ und selbstverständlich angenommen oder nicht gezählt werden, tauchen diese in den meisten Theorien über Wirtschaft nicht auf.

Doch was bedeutet "Care-Ökonomie“ beispielsweise in Deutschland in Zahlen? 2017 lag die geschätzte Wertschöpfung der unbezahlten Hausarbeit bei 1.000 Milliarden Euro Im Vergleich dazu lag die Wertschöpfung des produzierenden Gewerbes bei 800 MilliardenEuro (BMBF 2017). Diese Zahlen bringen die Spannbreite der sogenannten „Care-Ökonomie“ prägnant auf den Punkt.

\section{Welche Fragen werden seitens der feministischen Ökonomie gestellt?}

Es gibt keine einheitliche Definition der feministischen Ökonomie. Gleichzeitig lassen sich übergreifende Ansatzpunkte definieren.

- Erforschung eingeschriebener Geschlechter- und Herrschaftsverhältnisse in ökonomischen Theorien in Wechselwirkung miteinander und auf allen Ebenen: Mikroebene (individuelle Verhaltensweisen, habitualisierte Machtverhältnisse, Verhalten in kleinen Gruppen), Mesoebene (Geschlechterordnung in allen Organisationsformen) und Makroebene (gesellschaftliche Prozesse),

- Infragestellung der herkömmlichen Lehrmeinung der Mainstream-Ökonomie und des Menschenbilds des Homo oeconomicus, also der rational und ungebunden agierenden Akteur/innen,

- Kritik an der Bemessung von Wirtschaftsleistung durch das Bruttoinlandsprodukt (BIP), Kritik an der Messbarkeit von Leistung, am Arbeitsbegriff und der Unterteilung zwischen Produktion/Reproduktion,

- Hinterfragen von Sinn und Zweck von Wirtschaftsprozessen; Kritik an der fehlenden Berücksichtigung von Grundlagen und Bedürfnissen der Menschen sowie Endlichkeit von Ressourcen; Forderung danach, die Bedürfnisse der Menschen in den Mittelpunkt zu stellen (Bauhard 2012),

- Hinterfragen der Machtverhältnisse im wissenschaftlichen Diskurs/Ranking von Theorien. 


\section{„Der Mythos des abgeschlossenen Marktes würde von der Versorgung durch Haushalte, Markt, Allmende und Staat abgelöst."}

Konsequent zusammenhängend gedacht stellt feministische Ökonomie systemkritische Fragen und deckt entsprechende Möglichkeiten auf, einen anderen Blick auf die Wirtschaftstheorien und entsprechende Politik zu entwickeln.

\section{Wachstumskritik und Nachhaltigkeit aus geschlechterpolitischer Perspektive}

Die Kritik am BIP als Maßstab von Wirtschaftsleistung ist fast so alt wie das BIP selbst. Seit dem Bericht des Club of Rome 1972 ist das Thema Wachstum eine heutzutage über die Expert/innenkreise hinaus anerkannte Fragestellung. Wachstumsdiskurse sind im sogenannten "Mainstream“ angekommen. Gerade nach der Finanzkrise äußerten sich viele Deutsche wachstumskritischer. Nur noch ein Drittel glaubte, dass Wachstum auch die private Lebensqualität erhöhen wird (Die Zeit 2010). Das Wachstumskonzept und die Art und Weise des Wirtschaftens waren von jeher ein Bestandteil der feministischen Diskurse. Dabei sind die Konzepte aus der feministischen Ökonomie vielfältig und reichhaltig, dementsprechend nicht in einen Satz zu fassen. Sie reichen vom Konzept des "vorsorgenden Wirtschaftens" (Netzwerk Vorsorgendes Wirtschaften 2018) über die international organisierten, bei den UN Konferenzen vorgestellten Ansätze des Sustainable Livelihood-Konzepts (vom DAWN Network maßgeblich vorangetrieben) (Wichterich, 2012) bis zu den Queer Ecologies (Gottschlich/ Katz 2016).

Hier ist es wichtig, Verknüpfungen und Querverbindungen zwischen den oben genannten Ansätzen und den institutionell geführten Initiativen und Diskursen herzustellen, die gerade nach der Finanzkrise verstärkt initiiert wurden. Regierungen riefen entsprechende Expert/innenkomissionen ins Leben. Es ging und geht um ein neues Konzept, an dem sich alle orientieren können, und darum, etwas zu ermöglichen, dass das BIP möglicherweise ablösen kann. 2008 hatte der damalige französische Präsident Nicolas Sarkozy die Stiglitz-Sen-Fitoussi-Kommission initiiert, die ein „Dashboard“ von zwölf Empfehlungen erarbeitet hatte. In diesen Empfehlungen finden sich einige der Forderungen wieder, die aus der feministischen Ökonomie immer wieder eingebracht wurden. Beispielsweise die Berücksichtigung der Perspektive der Haushalte, einen anderen
Blick auf das „well being“, Verteilungsgerechtigkeit und vieles mehr (Stiglitz-Sen-Fitoussi 2010). Auf europäischer Ebene haben die Ergebnisse zu einigen Initiativen und neuen Empfehlungen geführt, die sich in der Bildung neuer Arbeitsgruppen und Berichten mit Empfehlungen für Umsetzungsmaßnahmen widerspiegeln. Die OECD gründetet die „Better Life Initiative“ mit der Veröffentlichung des Berichts „How's life? Measuring Well-Being" (OECD, 2011).

Die Handschrift der feministischen Ökonomie ist da, ohne dass sie immer explizit genannt wird (Biesecker et al. 2012). Auch der 17. Bundestag richtete 2010 eine Enquete Kommission zu Wachstum, Wohlstand, Lebensqualität ein. Sie erarbeitete einen Indikatorensatz, der über das BIP hinausgehend Wohlstand und Fortschritt messen soll. Weibliche Sachverständige in den Beirat einzubeziehen sowie die Diskussion um Integration feministischer Ökonomie hat hier für viel Furore gesorgt (Gersmann, 2011). Seit dem Abschlussbericht 2013 ist es still um dieses Thema in Deutschland geworden, obwohl es an Dringlichkeit nicht verloren hat. Überblickend beinhalten die einzelnen Initiativen und Berichte mehr oder minder starke Referenzen auf die feministische Ökonomie. Um entsprechende Anhaltspunkte zu vertiefen oder neue Vorschläge zu erarbeiten, müssen im Detail weitere Leerstellen aufgedeckt werden.

Zusammenfassend lässt sich in all den Initiativen, Kommissionen und Ausschüssen ablesen, dass ein neues ökonomisches Narrativ überfällig ist. Da in der feministischen Ökonomie oft interdisziplinär gearbeitet wird, ist die Veröffentlichung der Donut Ökonomie von Kate Raworth ein Ansatz, die diversen Stränge zusammenzudenken (Raworth 2018). Sie ist Ökonomin und hat aus 25 Jahren Forschung ein neues Bild entworfen. Es geht um ein Gesamtbild und nicht nur um Querverweise. Eine alte Kritik der feministischen Ökonomie ist, dass es nicht um das Hinzuaddieren von Geschlechterkategorien geht, sondern um das selbstverständliche Integrieren dieser Ansätze von vornherein in ein Konzept. Raworth bezieht in ihrem Ansatz Aspekte aus der feministischen Ökonomie selbstverständlich ein. Sie entwickelt einen interdisziplinären Ansatz, der als Ergebnis ein ökonomisches Narrativ entwirft, das sie als „Donut" zusammenfasst. Das soziale Fundament bildet die Grundlage des Lebens und die ökologische Decke ist die äußere Grenze. In diesem Rahmen sollte gewirtschaftet werden.

\section{Ein neues ökonomisches Narrativ ist überfällig}

Dabei enthält das soziale Fundament zwölf Dimensionen, die aus den Zielen für nachhaltige Entwicklung abgeleitet wurden (Sustainable Development Goals). Deswegen ist es für sie beispielsweise selbstverständlich, in einem ihrer sieben unterschiedlichen Denkstränge von einer eingebetteten Ökonomie auszugehen. Der Mythos des abgeschlossenen Marktes würde von der Versorgung durch Haushalte, Markt, Allmende und Staat abgelöst, die alle in die Gesellschaft und in die lebendige Welt eingebunden sind. Für sie ist der private Haushalt die 
Kernwirtschaft, von der alles abhängt (Raworth 2018). In dem Grundgerüst ihres Konzeptes lässt sich vieles aus unterschiedlichen Denkschulen der feministischen Ökonomie wiederfinden. Von der Kritik an der Verwendung des BIP als Maßstab, über eine eingebettete Ökonomie, dem Inkludieren des sozialen Wesens Mensch über Verteilungsgerechtigkeit und „eine(r) agnostische(n) Haltung zum Wachstum“ lassen sich Querverbindungen $\mathrm{zu}$ unterschiedlichen Denkschulen aus der feministischen Theorie herstellen - interdisziplinär gedacht und ausformuliert in ein Konzept und Bild. Das ist ein Schritt, der sich aus alten Mustern löst und den Mut hat, einen ganz neuen Entwurf zu starten. Das ist Zukunftsmusik - denn Krisen haben wir genug durch die alten Denkweisen produziert. Es bedarf neuer Konzepte, um Lösungen zu finden!

\section{Literatur}

Bauhardt, C. (2012): Feministische Ökonomie, Ökofeminismus und Queer Ecologies. Feministisch-materialistische Perspektiven auf gesellschaftliche Naturverhältnisse. www.fu-berlin.de/sites/gpo/pol_theorie/ Zeitgenoessische_ansaetze/Bauhardtfemoekonomie/Bauhardt.pdf Biesecker, A./Wichterich, C./Winterfeld, U. von (2012): Feministische Perspektiven zum Themenbereich Wachstum, Wohlstand, Lebensqualität. Hintergrundpapier. Berlin: Rosa-Luxemburg-Stiftung. Bremen, Bonn, Wuppertal: Rosa-Luxemburg-Stiftung.

Bundesministerium für Familie, Senioren, Frauen und Jugend (BMBF) (2017): Zweiter Gleichstellungsbericht der Bundesregierung.

Gersmann, H. (2011): Politik verlässt sich auf einen einseitigen Männerclub. In: taz 14.02. 2011, 6.

Gottschlich, D./Katz, C. (2016): Sozial-ökologische Transformation braucht Kritik an den gesellschaftlichen Naturverhältnissen. Zur notwendigen Verankerung von Nachhaltigkeitsforschung in feministischer Theorie und Praxis. In: Soziologie und Nachhaltigkeit. Beiträge zur sozial-ökologischen Transformationsforschung (SuN) 3/2016, 2. Jahrgang.

Keen, S. (2011): Debunking Economics. The Naked Emperor Dethroned? London, Zed Books.

Marçal, K. (2016): Who cooked Adam Smith's Dinner? A Story About Women and Economics. New York, Pegasus Books.
Netzwerk Vorsorgendes Wirtschaften (2018). http://www.vorsorgendes wirtschaften.de

OECD (2011): Better Life Initiative: Measuring Well-Being and Progress. www.oecd.org/statistics/better-life-initiative.htm

Pinzler, P. (2010): Wachstumsskeptisch. Eine Umfrage zeigt: Die Deutschen zweifeln am Kapitalismus. In: DIE ZEIT 19.08. 2010. (Basierend auf Ergebnissen des Meinungsforschungsinstituts Emnid im Auftrag der Bertelsmann-Stiftung.)

Ratzesberger, P. (2015): Mehr Vielfalt in der Ökonomie Tschüss, Neoklassik! In: Süddeutsche Zeitung 30.06. 2015.

Raworth, K. (2018): Die Donut-Ökonomie. Endlich ein Wirtschaftsmodell, das den Planeten nicht zerstört. München, Hanser.

Stiglitz, J./Sen, A./Fitoussi, J. (2010): Report by the Commission on the Measurement of Economic Performance and Social Progress. Im Auftrag der Europäischen Kommission.

Wichterich, C. (2012): Die Zukunft, die wir wollen. Eine feministische Perspektive. In: Heinrich-Böll-Stiftung (Hrsg.): Schriftenreihe zur Ökologie, Band 21.

\section{AUTORIN + KONTAKT}

Deborah Ruggieri ist Kultur- und Politikwissenschaftlerin (M. A.) und arbeitet seit acht Jahren selbstständig als Beraterin, Trainerin, Coach und als Wissenschaftlerin zu den Themen internationale Wirtschafts- und Finanzmarktpolitik\& Green Economy aus Geschlechterperspektive, Gleichstellungspolitik.

Deborah Ruggieri, ... communication rules!, Boxhagener Str. 65, 10245 Berlin. Tel.: +49 3091593887. E-Mail: contact@deborah-ruggieri.de, Website: www.deborah-ruggieri.de.

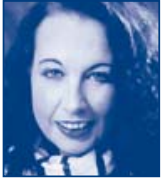

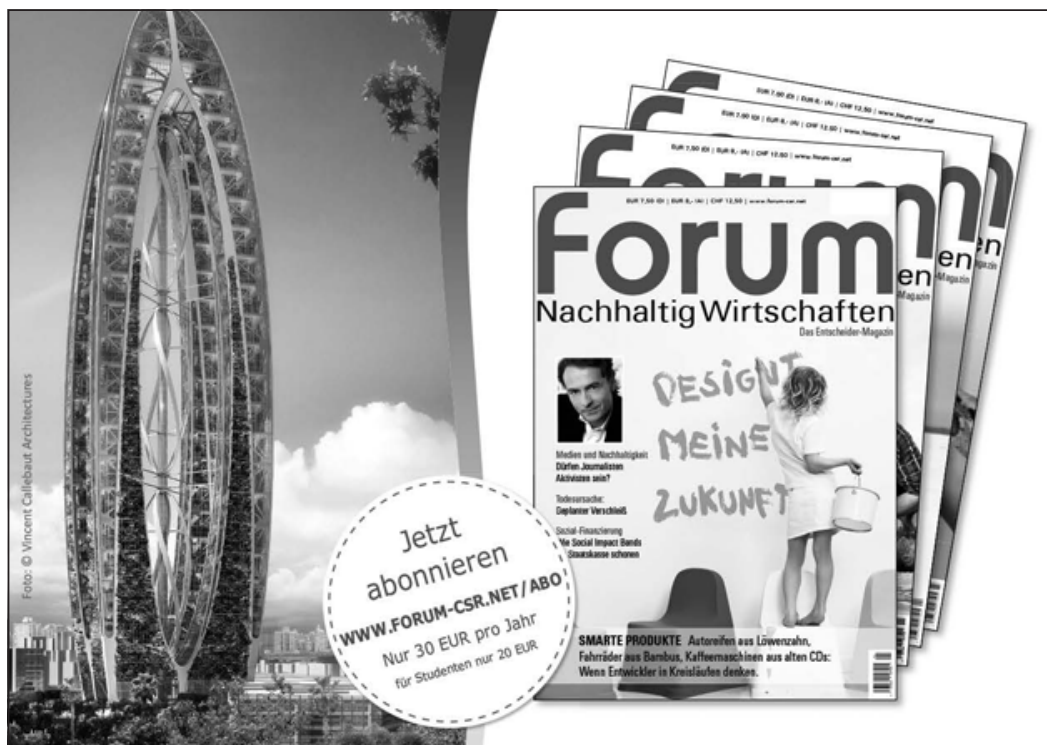

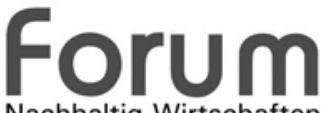

Nachhaltig Wirtschaften

\section{Zukunft gestalten}

Top informiert mit dem EntscheiderMagazin für nachhaltiges Wirtschaften und Corporate Social Responsibility!

Jetzt für 7,50 EUR am Kiosk oder unter www.forum-csr.net/Abo.

Auch als e-Magazin oder PDF.

Tel.: +49 (0)89 / 746611 - 10

Mail: abo@forum-csr.net 